\title{
Extra-hepatic comorbidity burden significantly increases 90 -day mortality in patients with cirrhosis and high model for endstage liver disease
}

Scott Coppel ${ }^{1}$, Karan Mathur ${ }^{2}$, Burcin Ekser ${ }^{3}$, Kavish R. Patidar², Eric Orman², Archita P. Desai², Eduardo Vilar-Gomez ${ }^{2}$, Chandrashekhar Kubal ${ }^{3}$, Naga Chalasani ${ }^{2}$, Lauren Nephew ${ }^{2 \dagger}$ and Marwan Ghabril ${ }^{2 * \dagger}$ (D)

\begin{abstract}
Background: We examined how extra-hepatic comorbidity burden impacts mortality in patients with cirrhosis referred for liver transplantation (LT).

Methods: Adults with cirrhosis evaluated for their first LT in 2012 were followed through their clinical course with last follow up in 2019. Extra-hepatic comorbidity burden was measured using the Charlson Comorbidity Index (CCI). The endpoints were 90-day transplant free survival (Cox-Proportional Hazard regression), and overall mortality (competing risk analysis).

Results: The study included 340 patients, mean age $56 \pm 11,63 \%$ male and MELD-Na 17.2 \pm 6.6. The CCI was 0 (no comorbidities) in 44\%, 1-2 in 44\% and $>2$ (highest decile) in 12\%, with no differences based on gender but higher CCl in patients with fatty and cryptogenic liver disease. Thirty-three (10\%) of 332 patients not receiving LT within 90 days died. Beyond MELD-Na, the CCI was independently associated with 90-day mortality (hazard ratio (HR), 1.32 (95\% confidence interval (CI) 1.02-1.72). Ninety-day mortality was specifically increased with higher CCl category and MELD $\geq 18(12 \%(C C l=0), 22 \%(C C l=1-2)$ and 33\% $(C \mathrm{Cl}>2),(p=0.002))$ but not MELD-Na $\leq 17$. At last followup, 69 patients were alive, 100 underwent LT and 171 died without LT. CCI was associated with increased overall mortality in the competing risk analysis (Sub-HR 1.24, 95\%Cl 1.1-1.4).

Conclusions: Extra-hepatic comorbidity burden significantly impacts short-term mortality in patients with cirrhosis and high MELD-Na. This has implications in determining urgency of LT and mortality models in cirrhosis and LT waitlisting, especially with an ageing population with increasing prevalence of fatty liver disease.
\end{abstract}

Keywords: Cirrhosis, Comorbidity, Charlson comorbidity index, Liver transplantation, Mortality

\footnotetext{
* Correspondence: mghabri|@iu.edu

${ }^{\dagger}$ Lauren Nephew and Marwan Ghabril share senior authorship

${ }^{2}$ Gastroenterology and Hepatology, Indiana University, 702 Rotary Circle, suite 225, Indianapolis, IN 46202, USA

Full list of author information is available at the end of the article
}

(c) The Author(s). 2020 Open Access This article is licensed under a Creative Commons Attribution 4.0 International License, which permits use, sharing, adaptation, distribution and reproduction in any medium or format, as long as you give appropriate credit to the original author(s) and the source, provide a link to the Creative Commons licence, and indicate if changes were made. The images or other third party material in this article are included in the article's Creative Commons licence, unless indicated otherwise in a credit line to the material. If material is not included in the article's Creative Commons licence and your intended use is not permitted by statutory regulation or exceeds the permitted use, you will need to obtain permission directly from the copyright holder. To view a copy of this licence, visit http://creativecommons.org/licenses/by/4.0/ The Creative Commons Public Domain Dedication waiver (http://creativecommons.org/publicdomain/zero/1.0/) applies to the data made available in this article, unless otherwise stated in a credit line to the data. 


\section{Background}

Cirrhosis is a serious consequence of chronic liver diseases, and represents a substantial burden of morbidity, mortality and health-care expenditure. It carries a poor prognosis in the setting of decompensation or development of hepatocellular carcinoma, with liver transplantation (LT) being the only definitive and lifesaving therapy. In this context, extra-hepatic comorbidities may carry multiple hazards to patients with cirrhosis in need of LT. They carry direct risk of mortality related to the impact of comorbidity [1, 2], as well as risk of precluding candidacy for lifesaving LT [3], and even risk of post LT mortality [4].

The Charlson Comorbidity Index (CCI) is a well described and validated instrument determined by the presence, and in some cases severity, of 16 comorbid conditions, including liver disease [5]. The CCI predicts 1 -year mortality in general populations and in patients with organ specific disease such as acute and chronic heart disease [5-8]. Both liver disease severity, reflected in MELD, and HCC are known to impact mortality and LT considerations in patients with cirrhosis. Beyond MELD, CCI predicts mortality in patients with suspected drug-induced liver injury [9]. However, the impact of extra-hepatic comorbidity burden on short-term and overall mortality in patients with advanced cirrhosis referred for LT has not been well-studied or quantified. The assessment of overall comorbidity burden, rather than individual comorbid conditions considered by transplant centers, may provide an aggregate measure of risk posed by the burden of extra-hepatic conditions.

In this study we measured the extra-hepatic medical comorbidity burden in a cohort of consecutive patients referred for LT using CCI (excluding the contributions of liver disease and HCC). The aims of the study were to determine the impact of extra-hepatic comorbidity burden on 90-day mortality and overall mortality in the study cohort.

\section{Patients and methods}

\section{Patients}

This study was approved by the Indiana University institutional review board, and was performed and reported per standardized reporting guidelines for qualitative research [10]. All patients with cirrhosis evaluated for LT at our center in 2012 were assessed. Patients were followed from the time of initial assessment through their pre and post-LT course until last follow-up in 2019. The selection of the study period was designed to allow for a relatively long post-LT follow-up (anticipated 5 years or more).

Patients with prior LT, absence of cirrhosis, or referred for multi-organ transplant were excluded. Demographic and clinical data were collected, including age, gender, body mass index (BMI), race, and etiology of liver disease. The severity of liver disease was measured using the MELD sodium equation (MELD-Na) [11]. Patients were followed until last contact for survivors or until death.

\section{Comorbidity burden}

Extra-hepatic comorbidity burden was measured using the CCI (contributions of liver disease and HCC to the malignancy component were excluded). For example, a patient with no extra-hepatic comorbidities but with cirrhosis and HCC would have a CCI of 0 . The CCI was chosen as an easily calculated and widely recognized comorbidity score that is validated in multiple populations including patients with chronic and acute liver disease $[5,9,12]$.

The CCI was analyzed primarily as a continuous variable in all risk-models. In addition, to examine the impact of low and high comorbidity burden we described outcomes within CCI categories using the following two thresholds; (i) patients with $\mathrm{CCI}=0$, a physiological reference group with the lowest extra-hepatic comorbidity burden, and (ii) the highest decile of CCI ( $>2$ in this cohort) to reflect the impact of the highest comorbidity burden. Patients with $\mathrm{CCI}=1-2$ represented an intermediate comorbidity burden group.

\section{Study endpoints}

The cohort was followed through the LT evaluation process with long-term follow up. The primary outcomes were 90-day (short-term) mortality without LT from the time of initial evaluation, which was examined in patients not undergoing LT within 90-days. The secondary outcome was overall mortality (longer-term) with $L T$ as a competing risk, which was examined in all patients.

\section{Additional analyses}

As a means of sensitivity testing for the association of comorbidity burden with mortality, we repeated all analyses while measuring comorbidities with an alternate score to CCI. The extra-hepatic comorbidity burden was measured and analyzed using the Cirrhosis Comorbidity score (CIRCOM), which has been validated in patients with cirrhosis [2]. Both CCI and CIRCOM are validated in patients with liver disease but they measure comorbidity burden somewhat differently. While CCI is a simple additive score, CIRCOM is designed as a conditional model based on priority scores and variable inclusion of specific groups of conditions. The individual comorbid conditions and their relative weights in CCI and CIRCOM are also not uniformly shared nor equally weighted (Supplemental Table 1). We examined the associations of the two scoring systems and their 
component conditions with short and long-term mortality in this cohort.

Finally, we examined the impact of extrahepatic comorbidity burden on LT eligibility after excluding patients with obvious prohibitive conditions and those deemed too early for or declining evaluation for LT.

\section{Statistical methods}

The analyses for factors associated with the study endpoints were performed using univariable and multivariable Cox proportional hazards regression for 90-day mortality, and univariable and multivariable competing risk regression for overall mortality, with $\mathrm{LT}$ as competing risk. The analyses were adjusted for age, gender, BMI, race, etiology of liver disease, MELD-Na and HCC. All analyses were two-sided with significance set at a $p$ value $<0.05$, and were performed using SPSS 26 (IBM Corp. Released 2017. IBM SPSS Statistics for Windows, Version 26.0. Armonk, NY: IBM Corp) or Stata SE 16 (StataCorp. 2019. Stata Statistical Software: Release 16. College Station, TX: StataCorp LLC).

\section{Results}

Among the 387 patients evaluated for LT 47 were excluded, including 30 patients who were referred for multi-organ transplant, 7 with prior LT and 10 without underlying cirrhosis. The remaining 340 patients met the inclusion criteria. Baseline demographic and clinic characteristics are summarized in Table 1 . The mean CCI was $1 \pm 1.2$ and the median CCI was 1 (Interquartile range (IQR) 0,1 ), with a score distribution of 0 in $44 \%$, 1 to 2 in $44 \%$, and $>2$ in $12 \%$ of patients. There were no differences in mean $\mathrm{CCI}$ in females and males, although females had a trend for higher frequency of connective tissue disease $(6.2 \%$ vs. $2.3 \%,(p=0.06))$. Mean CCI and patient age differed significantly according to etiology of cirrhosis (fatty liver (1.3 \pm 1.1 and $61 \pm 8$ ), cryptogenic $(0.9 \pm 1.4$ and $62 \pm 8)$, viral $(1.1 \pm 1.3$ and $57 \pm 8)$, alcohol with viral (1.1 \pm 1.4 and $54 \pm 7)$, alcohol $(0.6 \pm 0.8$ and $53 \pm 10)$ and autoimmune $(0.5 \pm 1.1$ and $51 \pm 15)$ disease $(p=0.003$ and $<0.001)$, respectively). While CCI did not correlate with patient age per se (Pearson coefficient $0.08,(p=0.15))$, mean age increased with higher CCI category $(55 \pm 10$ with $\mathrm{CCI}=0,56 \pm 9$ with $\mathrm{CCI}=1-2$ and $59 \pm 7$ with $\mathrm{CCI}>2)(p=0.025)$.

\section{The impact of comorbidity burden on 90-day survival}

Among the 340 patients evaluated 33 died within 90 days without LT, while 8 underwent LT within 90-days and were excluded from this specific analysis. Causes of death included multiorgan failure (7), infection (4), gastrointestinal bleed (4), cardiac (3), stroke (1) and undetermined (14). The CCI was independently associated with increased 90-day mortality on multivariable Cox regression analysis (Table 2), as was MELD-Na. The factors not associated with 90-day mortality included age, gender, BMI, race, etiology of liver disease and HCC. The results were similar when excluding 7 patients with moderate to severe renal disease (contributing to $\mathrm{CCI}$ ) which also contributed to higher MELD-Na. Extrahepatic comorbidity burden as measured by CIRCOM was associated with an almost identical 90-day mortality risk (adjusted HR 1.34, 95\%CI 1.02-1.77).

On closer examination, the impact of CCI on 90-day mortality was largely related to increasing risk in patients with MELD-Na above the median value of 17 . In patients with MELD-Na $\geq 18$, 90-day mortality was $12 \%$ with $\mathrm{CCI}=0,22 \%$ with $\mathrm{CCI}=1-2$ and $33 \%$ with $\mathrm{CCI}>2$, $(p=0.03))$. Whereas in patients with MELD-Na $\leq 17,90$ day mortality was $1 \%$ with $\mathrm{CCI}=0,1 \%$ with $\mathrm{CCI}=1-2$ and $4 \%$ with $\mathrm{CCI}>2,(p=0.5))$. Patients with MELD$\mathrm{Na} \geq 18$ also had increasing 90-day mortality with higher categories of CIRCOM (11\% with CIRCOM $=0,18 \%$ with CIRCOM $=1-2$ and $39 \%$ with CIRCM $>2$ (highest decile for CIRCOM), $(p=0.002))$.

\section{The impact of comorbidity burden on overall survival}

The median overall follow-up to time of death, LT or last follow-up was 332 days (IQR 161, 919). During this time 186 patient died with a median time to death of 303 days (IQR 126, 822). The median follow-up in 54 patients alive at last contact without LT was 6 years (IQR $0.6,6.5)$, and in 100 patients who underwent LT was 5.7 years (IQR 4, 7.3). Post-LT patient and graft survival rates were both $90 \%$ at 1 year and $81 \%$ at 5 years. Patients who died without LT had higher comorbidity burden than those who survived or underwent LT (Table 3). Compared to surviving patients, they were also more commonly male with viral liver disease, higher MELD-Na and HCC.

The CCI was associated with increased overall mortality on multivariable competing risk regression analysis (Table 4). The risk-adjusted cumulative incidence of mortality increased with each CCI point (Fig. 1). Extrahepatic comorbidity burden as measured by CIRCOM was also associated with overall mortality (adjusted SubHR 1.3, 95\%CI 1.2-1.5). The CCI was associated with overall mortality irrespective of baseline MELD-Na (adjusted sub-HR 1.3 (95\%CI 1.1-1.6) with MELD-Na $\leq 17$, and adjusted sub-HR 1.2 (95\%CI 1.1-1.4) with MELD$\mathrm{Na} \geq 18$ ).

\section{Individual comorbidities versus comorbidity burden and mortality}

We examined the association of individual components of the CCI and CIRCOM with 90-day and overall mortality using univariable models due to small numbers of patients with each condition (Table 5). Only renal disease defined by a creatinine $\geq 1.5 \mathrm{mg} / \mathrm{dL}$ (included in 
Table 1 Baseline demographic and clinical characteristics of the 340 patients evaluated for liver transplantation for complications of cirrhosis. Data are shown as mean \pm standard deviation or percentage unless otherwise specified. Patients who died within 90 -days without LT and those who survived at least 90-days without LT are compared (8 patients underwent LT within 90-days and were excluded)

\begin{tabular}{|c|c|c|c|c|}
\hline $\begin{array}{l}\text { Demographic and clinical } \\
\text { characteristics }\end{array}$ & $\begin{array}{l}\text { Patients assessed } \\
N=340\end{array}$ & $\begin{array}{l}\text { Patients who died } \\
\text { within } 90 \text { days } \\
n=33\end{array}$ & $\begin{array}{l}\text { Patients who survived } \\
\text { 90-days without LT } \\
n=297\end{array}$ & $p$-value \\
\hline Age & $56 \pm 11$ & $57 \pm 9$ & $56 \pm 9$ & 0.5 \\
\hline Gender (male) (\%) & 63 & 21 & 40 & 0.04 \\
\hline \multicolumn{5}{|l|}{ Race (\%) } \\
\hline White & 90 & 85 & 91 & \multirow[t]{5}{*}{0.6} \\
\hline Black & 5 & 9 & 4 & \\
\hline Hispanic & 3 & 6 & 3 & \\
\hline Asian & 1 & None & 1 & \\
\hline Other & 1 & None & 1 & \\
\hline Body mass index & $30 \pm 7$ & $30 \pm 8$ & $30 \pm 6$ & 0.7 \\
\hline \multicolumn{5}{|l|}{ Etiology of liver disease (\%) } \\
\hline Alcohol & 19 & 12 & 20 & \multirow[t]{6}{*}{0.4} \\
\hline Alcohol and viral & 16 & 24 & 16 & \\
\hline Viral & 32 & 39 & 31 & \\
\hline Autoimmune & 8 & None & 8 & \\
\hline Fatty liver & 21 & 21 & 21 & \\
\hline Cryptogenic & 2 & 3 & 2 & \\
\hline MELD-Na & $17.2 \pm 6.6$ & $25.1 \pm 7.3$ & $16.2 \pm 5.8$ & $<0.001$ \\
\hline Hepatocellular carcinoma (\%) & 24 & 30 & 23 & 0.4 \\
\hline $\mathrm{CCl}$ (mean) & $1 \pm 1.2$ & $1.4 \pm 1.2$ & $1 \pm 1.2$ & 0.09 \\
\hline (median (IQR)) & $1(0,1)$ & $1(0,2)$ & $1(0,1)$ & \\
\hline \multicolumn{5}{|l|}{ CCl category (\%) } \\
\hline $\mathrm{CCl}=0$ & 44 & 30 & 46 & \multirow[t]{3}{*}{0.07} \\
\hline $\mathrm{CCl}=1-2$ & 44 & 52 & 43 & \\
\hline $\mathrm{CCl}>2$ & 12 & 18 & 110. & \\
\hline CIRCOM (mean) & $0.8 \pm 1.2$ & $1.6 \pm 1.4$ & $0 . \pm 1.1$ & 0.001 \\
\hline (median (IQR)) & $0(0,1)$ & $1(0,3)$ & $0(0,1)$ & \\
\hline
\end{tabular}

Abbreviations: CCI Charlson Comorbidity Index (excluding liver disease and liver cancer), IQR interquartile range, $L T$ liver transplantation, MELD-Na Model for Endstage Liver Disease with sodium modification

CIRCOM and contributes to MELD-Na) was associated with 90-day mortality. The conditions common to CCI and CIRCOM that were associated with overall mortality included congestive heart failure, renal disease and metastatic malignancy. The conditions included in CCI, but not CIRCOM, that were associated with overall mortality included chronic obstructive pulmonary disease, diabetes mellitus with complications, cerebrovascular disease, connective tissue disease and acquired immune deficiency syndrome. At least one of these conditions was observed in $27.1 \%$ of patients. The condition included in CIRCOM, but not CCI, that was associated with overall mortality was substance abuse (excluding alcohol) observed in $19.6 \%$ of patients.

\section{The impact of comorbidity burden on liver transplant} eligibility

After the initial visit, 40 patients were deemed too early for LT and 38 patients chose not to pursue LT, and did not complete testing. Half of these patients were female, with mean MELD $<15$, mean $\mathrm{CCI}<1$, and $<10 \%$ had HCC (Supplemental Table 2). Additionally, 48 patients died before completing transplant evaluation and, $28 \mathrm{did}$ not complete evaluation. These patients were more frequently male, with mean MELD> 15 and mean CCI > 1 (Supplemental Table 2). The most common barriers for LT eligibility in patients attempting but not completing LT evaluation were advanced HCC, morbid obesity and active substance abuse which are not reflected in CCI, 
Table 2 The analysis of factors associated with 90-day mortality without liver transplant (LT) by Cox regression in 332 patients with cirrhosis referred for LT, 33 of whom died within 90 days of initial evaluation. Eight of 340 patients assessed underwent LT within $90-$ days from initial assessment and were excluded from this analysis

\begin{tabular}{|c|c|c|c|c|c|c|}
\hline \multirow[t]{2}{*}{ Factor } & \multicolumn{3}{|c|}{ Univariable analysis } & \multicolumn{3}{|c|}{ Multivariable analysis } \\
\hline & Hazard ratio & $95 \%$ confidence interval & $p$-value & Hazard ratio & $95 \%$ confidence interval & $P$-value \\
\hline $\mathrm{CCl}$ & 1.2 & $(0.97-1.5)$ & 0.09 & 1.33 & $(1.02-1.73)$ & 0.037 \\
\hline MELD-Na & 1.22 & $(1.16-1.29)$ & $<0.001$ & 1.26 & $(1.18-1.33)$ & $<0.001$ \\
\hline Age & 1.01 & $(0.98-1.06)$ & 0.5 & 1.02 & $(0.96-1.07)$ & 0.5 \\
\hline Male gender & 2.4 & $(1.03-5.4)$ & 0.043 & 1.2 & $(0.5-3)$ & 0.6 \\
\hline Body mass index & 1.01 & $(0.96-1.07)$ & 0.4 & 1.02 & $(0.96-1.09)$ & 0.4 \\
\hline \multicolumn{7}{|l|}{ Race (reference white) } \\
\hline Black & 2.2 & $(0.7-7.1)$ & 0.2 & 2.9 & $(0.8-11)$ & 0.12 \\
\hline Hispanic & 1.9 & $(0.5-7.9)$ & 0.4 & 0.4 & $(0.1-1.7)$ & 0.19 \\
\hline Asian & $N A^{a}$ & & & $N A^{a}$ & & \\
\hline \multicolumn{7}{|c|}{ Etiology liver disease (Reference alcohol) } \\
\hline Alcohol and viral & 2.4 & $(0.7-8)$ & 0.15 & 2.8 & $(0.8-9.8)$ & 0.08 \\
\hline Viral & 1.9 & $(0.6-6)$ & 0.24 & 2.3 & $(0.7-7.8)$ & 0.15 \\
\hline Autoimmune & $N A^{a}$ & & & $N A^{a}$ & & \\
\hline Fatty liver & 1.6 & $(0.5-5.5)$ & 0.4 & 3.3 & $(0.8-14.3)$ & 0.11 \\
\hline Cryptogenic & 2.1 & $(0.2-18.8)$ & 0.5 & 2.6 & $(0.2-31)$ & 0.5 \\
\hline Hepatocellular carcinoma & 1.4 & $(0.7-2.9)$ & 0.4 & 2 & $(0.8-5)$ & 0.11 \\
\hline
\end{tabular}

Abbreviations: CCI Charlson Comorbidity Index (excluding liver disease and liver cancer), MELD-Na Model for End-stage Liver Disease with sodium modification, NA not applicable

Footnotes: ${ }^{a}$ no patients in the analysis or with the endpoint to analyze

although substance abuse is included in CIRCOM (Supplemental Table 3).

One hundred and seventy-seven patients completed the evaluation process and were discussed formally in the transplant selection committee. They were predominantly male (69\%), with mean baseline MELD $17 \pm 5.8$, mean CCI $0.98 \pm 1.26$, and $31 \%$ had HCC (Supplemental Table 2). Of these 177 comprehensively evaluated patients 120 were approved for listing by the transplant selection committee and 57 were deemed ineligible. The CCI was inversely associated with LT eligibility in the risk-adjusted model, whereas male gender and autoimmune liver disease were associated with LT eligibility (Table 6). Factors not associated with LT eligibility included age, MELD, BMI, race, and HCC. Extra-hepatic comorbidity burden was also inversely associated with LT-eligibility in the risk-adjusted analysis when assessed using CIRCOM (adjusted HR 0.74, 95\%CI 0.6-0.91, $p=$ 0.004).

The most common barriers to LT eligibility in the 57 patients were cardiac, advanced HCC, psychosocial concerns and debilitation (Supplemental Table 3). Cardiac factors prohibitive of LT in 16 patients were related to coronary artery disease in 13 and uncontrolled arrhythmias in 2, neither of which is reflected in CCI or CIRCOM. The individual comorbid conditions that impacted LT eligibility in the unadjusted analysis included coronary artery disease without infarction, congestive heart failure, peptic ulcer disease, diabetes with complication, renal disease and substance abuse (Supplemental Table 4). Only 120 patients were waitlisted of whom 100 underwent LT. Both CCI and CIRCOM did not impact waitlist or postLT survival in the risk adjusted analyses, although the number of patients analyzed was small (data not shown).

\section{Discussion}

The main novel finding in this study was that extrahepatic comorbidity burden adversely impacted 90-day mortality among patients with cirrhosis evaluated for LT. This interplay between CCI and MELD-Na for short-term mortality was largely attributed to increased risk in patients with MELD-Na $\geq 18$. Interestingly, the inflection threshold for improving survival benefit for LT in cirrhosis was recently demonstrated at a MELDNa range of 18-20 [13]. In other words, extra-hepatic comorbidity burden appears to amplify short-term mortality in patients with cirrhosis who benefit the most from LT, and may be an important consideration for LT urgency in these patients.

The association of CCI and 90-day mortality persisted even when excluding patients with moderate to severe renal disease that may confound the association due to contribution of creatinine to MELD-Na. Beyond renal dysfunction, which is already reflected in higher MELD- 
Table 3 Baseline demographic and clinical characteristics of the patients evaluated for liver transplantation (LT) for complications of cirrhosis among patients who; (i) were alive at last follow up without LT, (ii) died without LT or (iii) underwent LT. Data are shown as mean \pm standard deviation or percentage

\begin{tabular}{|c|c|c|c|c|}
\hline Demographic and clinical characteristics & $\begin{array}{l}\text { Survived without LT } \\
n=54\end{array}$ & $\begin{array}{l}\text { Died without LT } \\
n=186\end{array}$ & $\begin{array}{l}\text { Underwent LT } \\
n=100^{\mathrm{a}}\end{array}$ & $P$-value \\
\hline Age & $54 \pm 10$ & $57 \pm 9$ & $56 \pm 10$ & 0.14 \\
\hline Gender (male) (\%) & 43 & 64 & 71 & 0.002 \\
\hline \multicolumn{5}{|l|}{ Race (\%) } \\
\hline White & 87 & 91 & 91 & \multirow[t]{5}{*}{0.4} \\
\hline Black & 4 & 4 & 6 & \\
\hline Hispanic & 6 & 4 & 1 & \\
\hline Asian & None & 1 & 1 & \\
\hline Other & 3 & None & 1 & \\
\hline Body mass index & $28 \pm 6$ & $30 \pm 7$ & $30 \pm 6$ & 0.4 \\
\hline \multicolumn{5}{|l|}{ Etiology of liver disease (\%) } \\
\hline Alcohol & 29 & 34 & 30 & \multirow[t]{6}{*}{0.001} \\
\hline Alcohol and viral & 12 & 21 & 10 & \\
\hline Viral & 29 & 18 & 13 & \\
\hline Autoimmune & 9 & 2 & 17 & \\
\hline Fatty liver & 19 & 20 & 24 & \\
\hline Cryptogenic & 2 & 2 & 3 & \\
\hline MELD & $13.9 \pm 4.4$ & $17.7 \pm 7.1$ & $18.1 \pm 5.9$ & $<0.001$ \\
\hline Hepatocellular carcinoma (\%) & 7 & 27 & 25 & 0.01 \\
\hline $\mathrm{CCl}$ (mean) & $0.6 \pm 0.9$ & $1.1 \pm 1.3$ & $0.6 \pm 0.8$ & $<0.001$ \\
\hline (median (IQR)) & $0(\operatorname{IQR~} 0,1)$ & $1(\mathrm{IQR} 0,2)$ & $0(\operatorname{lQR} 0,1)$ & \\
\hline \multicolumn{5}{|l|}{ CCl Category (\%) } \\
\hline $\mathrm{CCl}=0$ & 59 & 34 & 56 & \multirow[t]{3}{*}{0.001} \\
\hline $\mathrm{CCl}=1-2$ & 33 & 50 & 38 & \\
\hline $\mathrm{CCl}>2$ & 8 & 16 & 6 & \\
\hline CIRCOM & $\begin{array}{l}0.6 \pm 0.8 \\
0(\operatorname{IQR} 0,1)\end{array}$ & $\begin{array}{l}1.1 \pm 1.3 \\
1(\operatorname{IQR} 0,2)\end{array}$ & $\begin{array}{l}0.4 \pm 0.9 \\
0(\operatorname{IQR} 0,1)\end{array}$ & $<0.001$ \\
\hline
\end{tabular}

Abbreviations: CCI cirrhosis-modified Charlson Comorbidity Index (excluding liver disease and liver cancer), IQR interquartile range, MELD-Na Model for End-stage Liver Disease with sodium modification

Footnotes: ${ }^{a}$ includes one patient who underwent LT at another center

$\mathrm{Na}$, no component conditions of CCI or CIRCOM were associated with 90-day mortality. Therefore, the overall burden of extrahepatic comorbidities, as measured by CCI or CIRCOM, rather than specific conditions were the drivers of risk for 90-day mortality. This underscores the potential utility of an aggregate comorbidity burden score, beyond individual conditions, in risk assessment for patients with advanced liver disease.

Liver transplantation was an important factor in the consideration of overall mortality, with expected high rates of patient survival at 5 years post LT. This necessitated the assessment of overall mortality with competingrisk regression as described by Fine and Gray [14]. Since few patients underwent LT within 90-days of initial evaluation the short-term mortality risk with CCI could not be attributed to the impact of $\mathrm{CCI}$ on transplant eligibility.
However, comorbidities may represent barriers for LT candidacy, and not surprisingly comorbidity burden was higher in patient who died compared with those undergoing LT or surviving without LT. This data suggests that comorbidity may carry dual risks for patients with cirrhosis. An increased short-term risk in those with more advanced disease, and longer-term risk of both mortality and potential barriers to life-saving LT as their liver disease detriorates $11-12 \%$.

The examination of the impact of individual comorbid conditions also demonstrated that no scoring system captured all conditions that appeared to impact overall mortality in the cohort. The CCI does not measure substance abuse which is included in CIRCOM, and CIR$\mathrm{COM}$ does not measure 5 conditions included in $\mathrm{CCI}$ (chronic obstructive pulmonary disease, diabetes mellitus 
Table 4 The factors associated with overall mortality in the competing-risk regression analysis with liver transplantation as the competing risk

\begin{tabular}{|c|c|c|c|c|c|c|}
\hline \multirow[t]{2}{*}{ Factor } & \multicolumn{3}{|c|}{ Univariable analysis } & \multicolumn{3}{|c|}{ Multivariable analysis } \\
\hline & Sub-Hazard ratio & 95\% confidence interval & $P$-value & Sub-Hazard ratio & 95\% confidence interval & $P$-value \\
\hline$\overline{\mathrm{CCl}}$ & 1.25 & $(1.14-1.38)$ & $<0.001$ & 1.24 & $(1.1-1.4)$ & $<0.001$ \\
\hline MELD-Na & 1.05 & $(1.02-1.08)$ & 0.002 & 1.05 & $(1.02-1.09)$ & 0.001 \\
\hline Age & 1.01 & $(0.99-1.02)$ & 0.2 & 1.01 & $(0.99-1.03)$ & 0.2 \\
\hline Male gender & 1.2 & $(0.9-1.6)$ & 0.3 & 0.94 & $(0.7-1.3)$ & 0.7 \\
\hline Body mass index & 1.01 & $(0.98-1.03)$ & 0.5 & 1.01 & $(0.98-1.03)$ & 0.7 \\
\hline \multicolumn{7}{|l|}{ Race (reference white) } \\
\hline Black & 0.9 & $(0.5-1.7)$ & 0.7 & 0.8 & $(0.4-1.7)$ & 0.5 \\
\hline Hispanic & 1.5 & $(0.7-3.3)$ & .3 & 1.4 & $(0.5-3.5)$ & 0.6 \\
\hline Asian & 1.3 & $(0.4-5)$ & 0.7 & 3.1 & $(1.6-5.7)$ & 0.001 \\
\hline \multicolumn{7}{|c|}{ Etiology liver disease (Reference alcohol) } \\
\hline Alcohol and viral & 1.7 & $(1.02-2.7)$ & 0.03 & 1.6 & $(0.96-2.67)$ & 0.07 \\
\hline Viral & 1.2 & $(0.8-1.8)$ & 0.4 & 1.1 & $(0.7-1.8)$ & 0.6 \\
\hline Autoimmune & 0.2 & $(0.08-0.6)$ & 0.006 & 0.2 & $(0.09-0.7)$ & 0.009 \\
\hline Fatty liver & 1.1 & $(0.7-1.7)$ & 0.7 & 0.9 & $(0.6-1.6)$ & 0.8 \\
\hline Cryptogenic & 1.1 & $(0.5-2.7)$ & 0.8 & 0.7 & $(0.4-2.2)$ & 0.8 \\
\hline Hepatocellular carcinoma & 1.3 & $(0.9-1.7)$ & 0.14 & 1.1 & $(0.7-1.6)$ & 0.7 \\
\hline
\end{tabular}

Abbreviations: CCI Charlson Comorbidity Index (excluding liver disease and liver cancer), MELD-Na Model for End-stage Liver Disease with sodium modification, NA not applicable

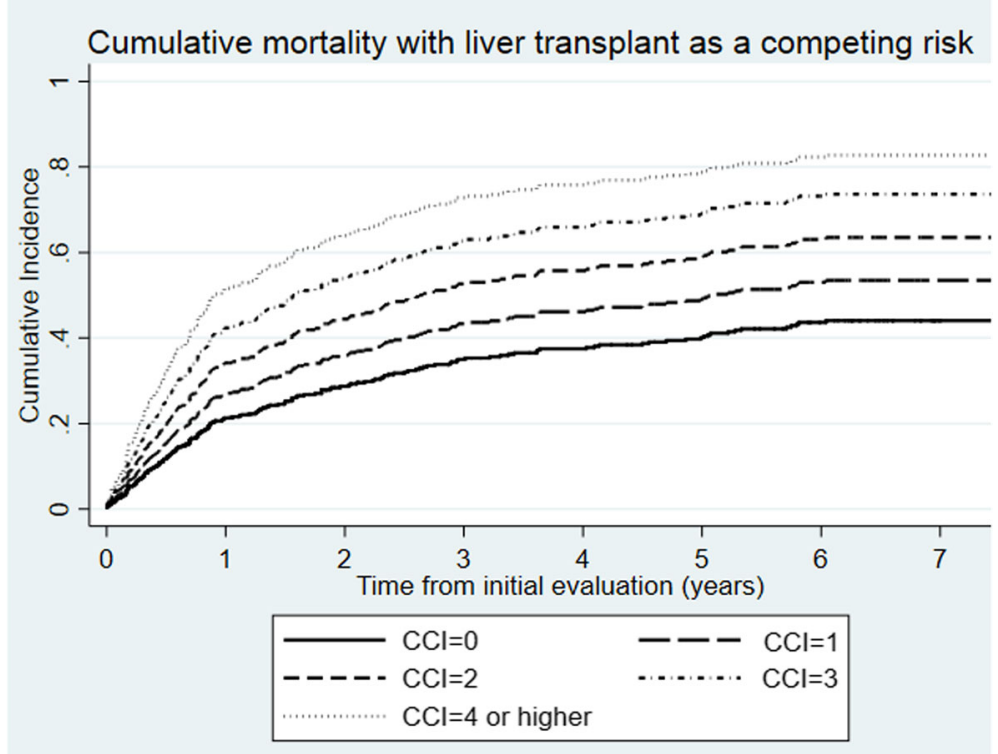

Fig. 1 Overall mortality in the competing risk model (liver transplant as the competing risk) stratified by comorbidity burden using the Charlson Comorbidity Index (CCI) (excluding liver disease and liver cancer). The analysis was adjusted for age, gender, race, model for endstage liver disease with sodium modification, body mass index, liver disease etiology and presence of hepatocellular carcinoma. The sub-hazard ratio for each 1-point increment in CCl was 1.24 (95\%confidence interval 1.1-1.4) 


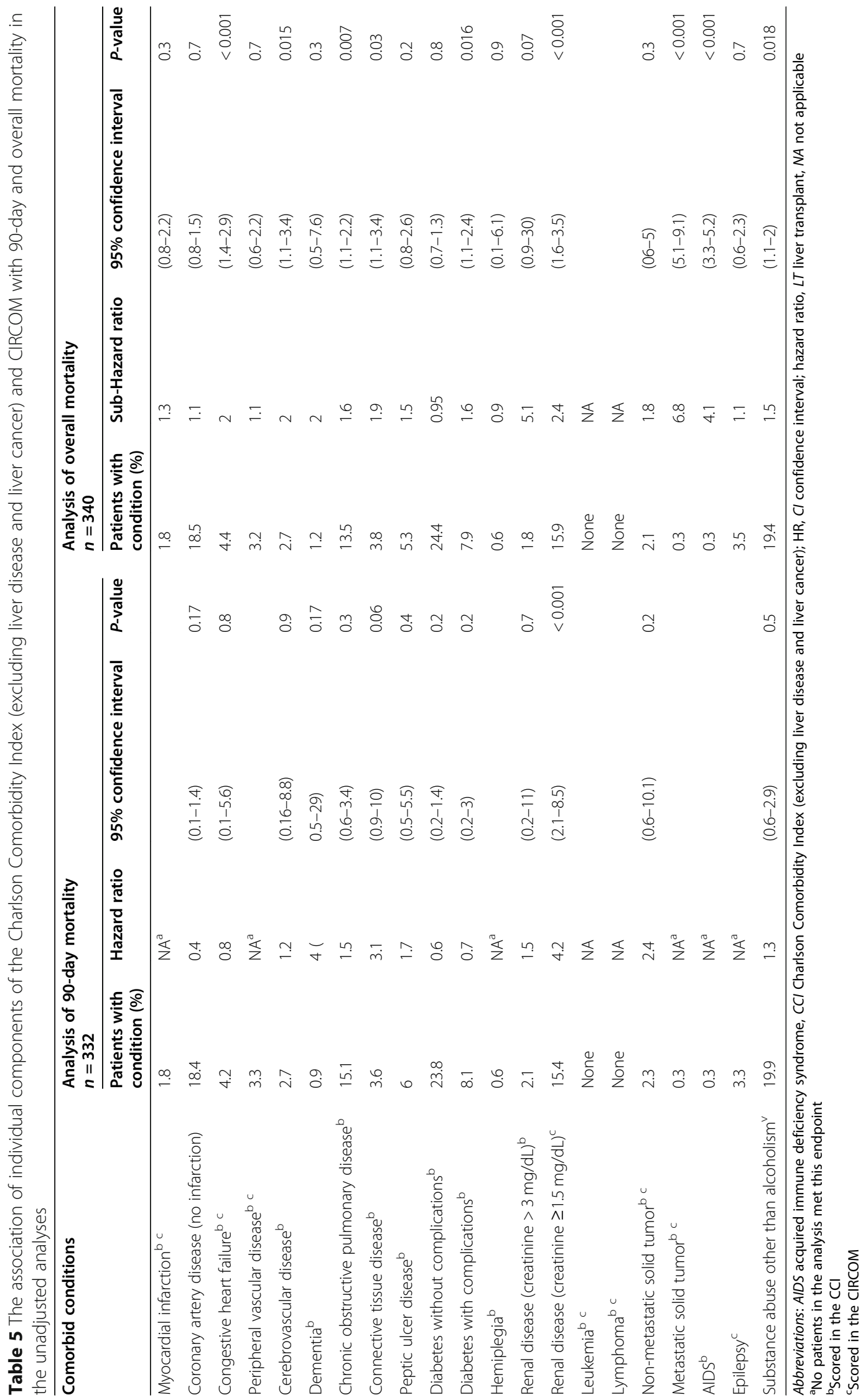


Table 6 The factors associated with liver transplant (LT) eligibility by Cox regression analysis in 177 patients completing work-up for LT

\begin{tabular}{|c|c|c|c|c|c|c|}
\hline \multirow[t]{2}{*}{ Factor } & \multicolumn{3}{|c|}{ Univariable analysis } & \multicolumn{3}{|c|}{ Multivariable analysis } \\
\hline & Hazard Ratio & $\mathbf{9 5 \%}$ confidence interval & $P$-value & Hazard Ratio & $95 \%$ confidence interval & $P$-value \\
\hline $\mathrm{CCl}$ & 0.64 & $(0.53-0.78)$ & $<0.001$ & 0.63 & $(0.51-0.79)$ & $<0.001$ \\
\hline MELD committee & 0.99 & $(0.96-1.01)$ & 0.2 & 1 & $(0.97-1.03)$ & 0.96 \\
\hline Age & 1.002 & $(0.98-1.025)$ & 0.9 & 1 & $(0.97-1.03)$ & 0.9 \\
\hline Male gender & 1.3 & $(0.9-1.9)$ & 0.16 & 2.3 & $(1.5-3.7)$ & $<0.001$ \\
\hline Body mass index & 0.98 & $(0.96-1.01)$ & 0.3 & 0.99 & $(0.96-1.03)$ & 0.7 \\
\hline \multicolumn{7}{|l|}{ Race (reference white) } \\
\hline Black & 0.71 & $(0.3-1.2)$ & 0.4 & 1.1 & $(0.4-2.6)$ & 0.9 \\
\hline Hispanic & 1.2 & $(0.4-3.8)$ & 0.8 & 1.8 & $(0.5-6.2)$ & 0.4 \\
\hline Asian & 4.6 & $(1.1-18.8)$ & 0.036 & 2 & $(0.5-10.6)$ & 0.4 \\
\hline \multicolumn{7}{|c|}{ Etiology of liver disease (Reference alcohol) } \\
\hline Alcohol and viral & 0.7 & $(0.3-1.3)$ & 0.2 & 0.6 & $(0.3-1.2)$ & 0.16 \\
\hline Viral & 1.1 & $(0.6-1.9)$ & 0.8 & 1.1 & $(0.6-2.1)$ & 0.7 \\
\hline Autoimmune & 2.1 & $(1.1-4)$ & 0.03 & 2.4 & $(1.2-5.2)$ & 0.02 \\
\hline Fatty liver & 1.1 & $(0.6-2)$ & 0.9 & 1.6 & $(0.8-3.2)$ & 0.17 \\
\hline Cryptogenic & 1.2 & $(0.4-3.7)$ & 0.7 & 0.9 & $(0.3-3.1)$ & 0.9 \\
\hline Hepatocellular carcinoma & 0.65 & $(0.43-0.98)$ & 0.038 & 1 & $(0.6-1.7)$ & 0.98 \\
\hline
\end{tabular}

Abbreviations: CCI Charlson Comorbidity Index (excluding liver disease and liver cancer), MELD Model for End-stage Liver Disease with sodium modification, NA not applicable

with complications, cerebrovascular disease, connective tissue disease and acquired immune deficiency syndrome). These differences would have impacted comorbidity burden measurement in 19.4 and $27.1 \%$ of patients, respectively. Both scores still performed well as measures of extra-hepatic comorbidity burden with very similar adjusted hazard and sub-hazard ratios. It is not within the scope nor the intent of this study to suggest modifications of comorbidity scoring systems, but we acknowledge the interesting differences between these scores that could impact 1 in 4 to 5 patients with advanced cirrhosis.

The CCI was developed as a continuous scale or variable and the categorical descriptive analysis warrants discussion. A CCI $=0$ reflects a physiologically important reference group with no or very low comorbidity burden. A CCI $>2$ highlighted the risks associated with the highest decile of extra-hepatic comorbidity burden, e.g. in patients with higher MELD-Na. Notably, other studies have demonstrated increased mortality in patients with cardiac disease and in patients without cirrhosis presenting with acute liver injury, using the same threshold [9, $15,16]$. However, the ideal thresholds if any for estimating CCI related risk in the context of pre-LT outcomes may be refined with additional studies.

A higher comorbidity burden was observed in patients with fatty and cryptogenic liver disease. The latter is commonly attributed to undiagnosed fatty liver disease, which is known to be associated with extra-hepatic comorbidities and increasingly driving the need for LT in the United
Sates $[17,18]$. While we observed age differences according to disease etiology, extra-hepatic comorbidity burden did not correlate with age per se, suggesting that the observed associations were mainly related to differences in disease associations rather than older age alone. Genderbased disparities in LT eligibility have been described with increased pre-LT and waitlist mortality in women $[19,20]$. Additionally, gender-based differences in medical comorbidities have been described in hospitalized patients with higher rates of diabetes and connective tissue disease but without evident differences in hospital mortality [21]. In our cohort, there were no gender-based differences in $\mathrm{CCI}$, although women had a trend for more frequent connective tissue disease. Higher 90-day mortality was observed in men, but that association was fully attenuated in the risk-adjusted analysis.

In practice, $\mathrm{LT}$ candidate selection is impacted by individual clinically relevant comorbidities and psychosocial considerations rather than a comorbidity burden score such as CCI. There is a dearth of data in this area, but Arya et al. have demonstrated that less than half of patients evaluated for LT were deemed ineligible, mainly for being too early, medical conditions or addiction problems [3]. We observed similar outcomes in our cohort and explored the role of comorbid conditions in detail as potential barriers to LT. Extra-hepatic comorbidity burden (CCI) was associated with LT-ineligibility and multiple comorbid conditions were individually associated with LT-ineligibility. However, the majority of barriers to 
LT in patients reviewed by the selection committee or in those not completing evaluation (coronary artery disease, obesity, being too ill or frail, advanced HCC or psychosocial issues such substance abuse) were not reflected in the CCI. Only 5 patients were LT-ineligible for other comorbidities (lung disease 2, and non-liver malignancy 3 ) which were reflected in CCI. The CCI components of diabetes with end organ damage and peptic ulcer disease were also associated with LT-ineligibility, but were not in of themselves contraindications for LT. In other words, conditions contraindicating LT were reflected in CCI in less than $10 \%$ of LT-ineligible patients. Therefore, measurement of extra-hepatic comorbidity burden was not a substitute for, but possibly complementary of, reasoned clinical judgment in determining LT candidacy.

The strengths of this study include the comprehensive characterization of comorbid conditions and the longterm follow up of patients with cirrhosis consecutively evaluated for LT. The assessment of extra-hepatic comorbidity burden using two validated systems (CCI and CIR$\mathrm{COM}$ ) and convergence of the observed associations also support the clinical premise of the study. The limitations of the study include the retrospective design, limited racial diversity, the absence of comprehensive information on socioeconomic status, and in some cases limited follow-up intervals in patients not undergoing LT. Finally, the use of $\mathrm{CCI}$ and CIRCOM in this study was demonstrative of the impact of comorbidity burden on mortality and was not a judgement of superiority of a specific score over other available instruments.

\section{Conclusion}

In summary, this study demonstrates and quantifies the risk associated with extra-hepatic comorbidity burden with increased short and long-term mortality in patients with advanced cirrhosis. These data are timely, given an ageing population and increasing burden of fatty liver disease and comorbid conditions on transplant and other healthcare resources. Assessment of comorbidity burden may identify a subset of patients with the highest mortality risk and increased LT urgency. If validated, standardized measurement of extra-hepatic comorbidity burden may also be an important modifier of mortality risk models and mortality-related healthcare metrics in cirrhosis. Further studies are needed to confirm these findings, and to determine the ideal comorbidity scoring system and thresholds in patients with advanced cirrhosis.

\section{Supplementary information}

Supplementary information accompanies this paper at https://doi.org/10. 1186/s12876-020-01448-z.

Additional file 1: Supplemental Table 1. The extra-hepatic conditions and their relative weights comprising the Charlson Comorbidity Index (excluding liver disease and hepatocellular carcinoma) and the cirrhosis comorbidity score (CIRCOM). Supplemental Table 2. Baseline demographic and clinical characteristics of the patients evaluated for liver transplantation (LT) for complications of cirrhosis among patients who; (i) were deemed too early for $L T$ (ii) did not want to pursue $L T$ (iii) did not complete LT evaluation (iv) died before competing LT evaluation, or (v) completed LT evaluation with selection committee review. Data are shown as mean \pm standard deviation or percentage. Supplemental Table 3. The barriers to liver transplant (LT) eligibility in 28 patients not completing LT evaluation and 57 patients completing evaluation but not approved for LT after selection committee review. Data shown as numbers (and percentages for the main categories of barriers). Supplemental Table 4. The univariable Cox regression analyses of association of individual components of the Charlson Comorbidity Index (excluding liver disease and liver cancer) and CIRCOM, with the study endpoints of liver transplant eligibility and post liver transplant survival.

\section{Abbreviations}

BMI: Body mass index; CCl: Charlson Comorbidity Index; CIRCOM: Cirrhosis Comorbidity score; Cl: Confidence interval; HCC: Hepatocellular carcinoma; HR: Hazard ratio; LT: Liver transplantation; MELD: Model for endstage liver disease; MELD-Na: Model for endstage liver disease with sodium modification

\section{Acknowledgements \\ None}

\section{Authors' contributions}

All authors have read and approved the manuscript, SC: Study design, data collection and analysis, manuscript preparation, final approval. KM: Data collection and analysis, manuscript preparation, final approval. LN: Study design, data collection and analysis, manuscript preparation, final approval. KRP: Study design, data collection and analysis, manuscript preparation, final approval. EO: Study design, data interpretation, manuscript preparation, final approval. APD: Data analysis, manuscript preparation, final approval. EVG: Data analysis, manuscript preparation, final approval. BE: Study design, data collection and analysis, manuscript preparation, final approval. CK: Study design, data collection and analysis, manuscript preparation, final approval. NC: Study design, data collection and analysis, manuscript preparation, final approval. MG: Study design, data collection and analysis, manuscript preparation, final approval.

\section{Funding}

This study was not funded.

\section{Availability of data and materials}

Per the Indiana University institutional review board, data is to be deidentified and stored on a secure server, but no permission to store on a publicly accessible server. Specific data points requests, excluding any patient identifiers, directed to the corresponding author will be considered and honored.

\section{Ethics approval and consent to participate}

This study was approved by the Indiana University institutional review board, and as a minimal risk study, consent to participate and publish was waived.

Consent for publication

The authors consent to the publication policy of the journal.

\section{Competing interests}

Dr. Chalasani holds consulting agreements from several pharmaceutical companies for activities related to NAFLD, DILI and liver disease in general. His institution receives research grants on his behalf from Intercept, Lilly, Cumberland, Galectin, and Exact Sciences. But none represent a conflict of interest for this paper. Scott Coppel, Karanm Mathur, Lauren Nephew, Kavish Patidar, Eric Orman, Archita Desai, Eduardo Vilar-Gomez, Burcin Ekser, Chandrashekhar Kubal, and Marwan Ghabril report no potential conflicts of interest. 


\section{Author details}

${ }^{1}$ Medicine, Indiana University, Indianapolis, IN, USA. ${ }^{2}$ Gastroenterology and Hepatology, Indiana University, 702 Rotary Circle, suite 225, Indianapolis, IN 46202, USA. ${ }^{3}$ Transplant Surgery, Indiana University, Indianapolis, IN, USA.

Received: 2 August 2020 Accepted: 11 September 2020

Published online: 16 September 2020

\section{References}

1. Bianchi G, Marchesini G, Zoli M, et al. Prognostic significance of diabetes in patients with cirrhosis. Hepatology. 1994;20:119-25.

2. Jepsen P, Vilstrup H, Lash TL. Development and validation of a comorbidity scoring system for patients with cirrhosis. Gastroenterology, 2014. 146:14756 quiz e15-6.

3. Arya A, Hernandez-Alejandro R, Marotta $\mathrm{P}$, et al. Recipient ineligibility after liver transplantation assessment: a single Centre experience. Can J Surg. 2013;56:E39-43.

4. Volk ML, Hernandez JC, Lok AS, et al. Modified Charlson comorbidity index for predicting survival after liver transplantation. Liver Transpl. 2007;13:1515-20.

5. Charlson ME, Pompei P, Ales KL, et al. A new method of classifying prognostic comorbidity in longitudinal studies: development and validation. J Chronic Dis. 1987:40:373-83.

6. Lee DS, Austin PC, Rouleau JL, et al. Predicting mortality among patients hospitalized for heart failure: derivation and validation of a clinical model. JAMA. 2003;290:2581-7.

7. Sanchis J, Nunez J, Bodi V, et al. Influence of comorbid conditions on oneyear outcomes in non-ST-segment elevation acute coronary syndrome. Mayo Clin Proc. 2011;86:291-6.

8. Sanchis J, Soler M, Nunez J, et al. Comorbidity assessment for mortality risk stratification in elderly patients with acute coronary syndrome. Eur J Intern Med. 2019;62:48-53.

9. Ghabril M, Gu J, Yoder L, et al. Development and validation of model consisting of comorbidity burden to calculate risk of death within 6 months for patients with suspected drug-induced liver injury. Gastroenterology. 2019; 157(5):1245-52.e3.

10. O'Brien BC, Harris IB, Beckman TJ, et al. Standards for reporting qualitative research: a synthesis of recommendations. Acad Med. 2014;89:1245-51.

11. Kim WR, Biggins SW, Kremers WK, et al. Hyponatremia and mortality among patients on the liver-transplant waiting list. N Engl J Med. 2008;359:1018-26.

12. Xu Y, Li N, Lu M, et al. Comparison of risk adjustment methods in patients with liver disease using electronic medical record data. BMC Gastroenterol. 2017:17:5.

13. Nagai $\mathrm{S}$, Chau LC, Schilke RE, et al. Effects of allocating livers for transplantation based on model for end-stage liver disease-sodium scores on patient outcomes. Gastroenterology. 2018;155:1451-62 e3.

14. Fine JP, Gray RJ. A proportional hazards model for the subdistribution of a competing risk. J Am Stat Assoc. 1999;94:496-509.

15. Formiga F, Moreno-Gonzalez R, Chivite D, et al. High comorbidity, measured by the Charlson comorbidity index, associates with higher 1-year mortality risks in elderly patients experiencing a first acute heart failure hospitalization. Aging Clin Exp Res. 2018;30:927-33.

16. Fraccaro $\mathrm{P}$, Kontopantelis $\mathrm{E}$, Sperrin $\mathrm{M}$, et al. Predicting mortality from change-over-time in the Charlson comorbidity index: a retrospective cohort study in a data-intensive UK health system. Medicine (Baltimore). 2016;95: e4973.

17. Pham T, Dick TB, Charlton MR. Nonalcoholic fatty liver disease and liver transplantation. Clin Liver Dis. 2016;20:403-17.

18. Wong RJ, Aguilar M, Cheung R, et al. Nonalcoholic steatohepatitis is the second leading etiology of liver disease among adults awaiting liver transplantation in the United States. Gastroenterology. 2015;148:547-55.

19. Moylan CA, Brady CW, Johnson JL, et al. Disparities in liver transplantation before and after introduction of the MELD score. JAMA. 2008;300:2371-8.

20. Cullaro G, Sarkar M, Lai JC. Sex-based disparities in delisting for being "too sick" for liver transplantation. Am J Transplant. 2018;18:1214-9.

21. Rubin JB, Srisengfa YT, Albhaisi S, et al. Clin Gastroenterol Hepatol. 2019; S1542-3565(19):31094-8

\section{Publisher's Note}

Springer Nature remains neutral with regard to jurisdictional claims in published maps and institutional affiliations.

\section{Ready to submit your research? Choose BMC and benefit from:}

- fast, convenient online submission

- thorough peer review by experienced researchers in your field

- rapid publication on acceptance

- support for research data, including large and complex data types

- gold Open Access which fosters wider collaboration and increased citations

- maximum visibility for your research: over $100 \mathrm{M}$ website views per year

At BMC, research is always in progress.

Learn more biomedcentral.com/submissions 